\title{
Research Review on Control Methods of Large-scale Electric Vehicles Charging Station Network
}

\author{
Qingsheng Shi* and Yifan Liu \\ College of Electrical Engineering, Henan University of Technology, Zhengzhou 450001, China \\ ${ }^{*}$ Corresponding author
}

\begin{abstract}
Large-scale operation of electric vehicle is the only way to speed up its industrialization, and charging station network is the vital link to electric vehicle's normal operation. Charging station network is a large, dynamic, and high degree of uncertainty distributed system. And it is influenced by many factors, such as charging mode, gird safety and land price. As the requirement of charging efficiency of station network becomes higher, it is important to achieve the optimal configuration and control of charging station network, while the systematic research of it is seldom. Therefore, the development status of electric vehicle charging station at home and abroad is analyzed followed by current research topics. Then, three key issues urgent to be solved are summarized during the electric vehicle largescale operation, and the corresponding solutions are also proposed.
\end{abstract}

Keywords - charging station network; electric vehicle; optimal configuration; optimal control

\section{INTRODUCTION}

In the 21st century, human faces enormous challenges in energy and environmental issues in sustainable transportation development. Traffic electrification is one of the most effective solutions to mitigate global warming and reduce pollution emissions. Therefore, the development of electric vehicles is not only conducive to energy-saving emission reduction and technological progress, but also an important measure for countries in the world to cope with the international financial crisis and accelerate the strategic transformation of transport energy [1-2]. After $R \& D$ in recent years, electric vehicles worldwide are at an important juncture in transition from R\&D to industrialization. At present, the cost and selling price of electric vehicles in the market introduction period are much higher than those of traditional cars, hindering market promotion. Therefore, various countries have carried out largescale demonstration operations of electric vehicles led by the government, cultivate the market, improve the industrial chain, reduce costs, and promote the industrialization of electric vehicles.

China has also proposed the "Made in China 2025" strategy for new energy vehicles and successively introduced the relevant policies of the Electric Vehicles and Charging Facilities industry, including the "General Office of the State Council on Accelerating the Promotion of New Energy Vehicles," to guide the healthy development of the electric vehicle and charging facilities industry. The "Electric Vehicle Recharging Infrastructure Development Guide (2015-2020)” plans to add more than 12,000 centralized charging and switching stations and more than 4.8 million decentralized charging stations in order to satisfy the demands of more than 2 million electric vehicles charging needs by2020. 2015 is the first year of the construction of charging facilities in China. There is a huge market for the construction of charging facilities in China. However, as China has just started the research on the large-scale operation of charging stations for electric vehicles, some key issues have not yet been resolved [3]. Therefore, in order to realize the economical and efficient operation of the charging station network, it is necessary to conduct in-depth research on its configuration and control issues. Therefore, this essay analyzes the status quo of the construction of charging stations and the main research contents, as well as presents several key problems that need to be solved in large-scale operations, also gives targeted solutions.

\section{CURRENT RESEARCH CONTENT OF ELECTRIC VEHICLE CHARGING STATIONS}

While the State Grid stepped up the construction of charging stations, many researchers also conducted research on some of the charging stations. Mainly concentrated in the following areas:

(1) Current status analysis of charging station function and construction progress [3-5]. For example, literature [3] analyzes the influencing factors that need to be considered in the related research of electric vehicles from the planning level and the operational level, and excavates the influencing factors with traffic attributes. In [4], a prototype of a home-use microcharging station was designed; in [5], the charging requirements of an electric vehicle were analyzed, and a preliminary study was made on the construction of a charging station from the perspective of external access to electrical energy.

(2) The potential harmonic effects of charging stations on the power grid and their governance issues [6-10]. For example, [6] briefly describes the impact of electric vehicle charging stations on the harmonics of power grids, as well as proposes an on-line power quality monitoring and harmonic management scheme for electric vehicle charging stations. The literature [7] uses the laws of probability and statistics to study multiple electric motors. The harmonic current and its probabilistic characteristics generated by the car charger on the power grid; literature[8] established a simulation model to analyze the harmonic current pollution power supply network 
and electric equipment problems of the pure electric bus charging station; literature [9] proposed a Simplified charging station harmonic engineering algorithm can calculate the harmonic variation characteristics and the harmonic maximum in a charging cycle. literature [10] analyzes the problems that the charging station may cause harmonics and voltage unbalance in the power grid.

(3) Research on the design of charging station management system [11][12-14]. For example, the literature [11] designed a charging station all-digital intelligent management information system to realize the timing storage of voltage, current and input/output energy during the charging process; [12] designed a management information system based on 485 bus and adopted the serial port. The data receiving and controlling of the charger have been realized. The literature [13] has designed and developed an EVCS2000 charging station monitoring system consisting of a basic platform, a supporting platform and an application system. The literature [14] introduces a method for the development of a database system. The charger parameter settings and charge and discharge control.

(4) Research on high power fast charging method of charging station. As shown in [15], using the energy storage buffer system to compensate the pulse power of the fast charging of the electric vehicle can reduce the adverse effect of the access of the fast charging station to the distribution network. The fast charging station structure with energy storage buffer system and the fast charging power characteristics of different types of batteries are studied. The operation mode and current control strategy of the energy storage buffer system that takes into account the active power rate variation limit of the distribution network are proposed.

(5) Research on location problem of electric vehicle charging station based on satisfactory optimization. As shown in [31], based on the theory of satisfactory optimization, an evaluation function of the user satisfaction of the electric vehicle is proposed; a multi-level electric vehicle charging station is established by introducing the concept of the charging station hierarchy and using the maximum satisfaction of the average electric vehicle user as the objective function. A mixed-integer programming model was selected and a model solving algorithm based on immune algorithm was proposed. Finally, it is verified that the model can effectively determine the location, grade and service area of the charging station, and the solution algorithm is fast and effective.

\section{Optimal AlLOCATION AND CONTROL OF ElECtRiC VEHICLE CHARGING STATIONS}

In summary, although people have done a lot of research on the charging station network, there are still some important issues that need to be solved: (1) How to achieve a large scale considering the number of vehicles, grid security and site costs The optimal configuration of the charging station network; (2) In order to ensure the supply of replaceable batteries in charging stations operating in mixed mode, how to achieve optimal delivery of replaceable batteries; (3) To avoid "some charge congestion between charging stations" In other cases, they are relatively idle. How to coordinate the power supply in the charging station is to optimize the control problem.
Based on a detailed analysis of the above issues, we will try to provide corresponding solutions.

\section{(1) Optimization of configuration problems}

For large-scale operation of electric vehicles, the configuration of the charging station is critical. Due to the high construction cost of the charging station, if there are too many charging stations, it will cause waste in resource allocation. If the number of charging stations is too small, it will cause the vehicle to wait too long and affect the normal operation of the vehicle. Therefore, the optimization of the number of charging stations can not only effectively reduce the cost of infrastructure construction, but also play a key role in reducing vehicle waiting time and improving the operating efficiency of vehicles. When configuring the number of charging stations, it is not possible to consider only the number of electric vehicles that are put into operation, but also factors such as power grid safety and site costs. In this way, the optimal configuration of the charging station in the charging station network can be attributed to a constrained nonlinear multi-objective optimization problem.

Secondly, because there are two ways of charging electricity and changing electricity in the current form of electric vehicle power supply, the charging load of electric vehicles currently has obvious time and space uncertainties. As a result, the battery change mode has become the energy supply mode that many users have chosen. For example, the literature [16] studied the problem of orderly charge control strategy for battery replacement in the power station, and analyzed the operation characteristics of the power station in an in-depth manner, and based on this, established an orderly battery charging model for the replacement station. The model proposed in the literature uses the number of battery charging inputs for each period of time as the control variable, comprehensively considers the substation capacity requirements of the substation, the satisfaction requirements of the substation, and the minimum squared deviation of the load curve. Secondly, this paper proposes an improved cuckoo algorithm to solve the integer programming problem, and uses this algorithm to achieve fast solution of the ordered charging model.

Recently, there have been only a few studies on the configuration of the charging station network. Literature [17] analyzed the charging requirements of electric vehicles by describing the operating modes and charging requirements of electric vehicles, and proposed that the development of electric vehicles is fast, universal, intelligent, and efficient in the conversion of charging technologies. The requirements of chemistry and other studies have compared the advantages and disadvantages of conventional charging modes such as conventional charging, fast charging, and mechanical charging. The principle of the number of charging stations for conventional charging and rapid charging is given, that is, under the normal charging mode, a charging station is configured with 20-40 electric vehicles. In the fast charging mode, a charging station is generally configured by charging 8 electric vehicles at the same time. This principle is relatively clear and simple, but there are no in-depth considerations and analyses of factors such as complete grid and site costs. If the 
optimal configuration of the charging station network is achieved, there must be many factors such as the number of vehicles, complete grid and site costs. However, these factors are mutually contradictory and can be solved with the aid of game theory. As an important branch of applied mathematics, game theory is the study of mathematical theories and methods that have phenomena of struggle or competition [18]. Game theory considers the individual's predicted behavior and actual behavior in the game and studies their optimization strategies. At present, the theory of game theory has been successfully used to solve the problem of dynamic resource allocation in multi-cell OFDMA systems [19] and the trust in the formation of enterprise R\&D alliances. Problem [20], Probabilistic Risk Analysis [21], etc. If a multi-player non-cooperative game model such as the number of charging stations, number of charging vehicles, power grid security and site cost can be established, the Bayesian equilibrium can be calculated by using game theory to achieve optimal configuration of the charging station network, and charging station optimization is given. The universal design principle of configuration.

\section{(2) Battery optimization distribution problem}

At present, there are two main types of electric car supply form in the world: direct charging of vehicles and battery replacement [4]. The direct charging of the vehicles in the charging station has a large instantaneous power, which will cause a large spike in the ordinary power grid and affect the safety of the power grid. The use of the evening valley battery charging the battery it can reduce the cost of charging, and can also achieve effective peaking of the power grid. Therefore, the use of direct charging and battery replacement is the most promising mode of operation for charging station construction. For a large-scale operating charging station network with a hybrid operating mode, only the availability of a relatively large number of replaceable batteries and charging spaces can be guaranteed. Can meet the charging requirements of the vehicle. The number of batteries that can be delivered in the entire charging station network is very large. Therefore, the timely and accurate delivery of a large number of replaceable batteries can not only ensure the normal operation of the entire charging station network, but also greatly reduce the manpower in the distribution process. Material costs.

In the field of battery distribution in charging stations, no relevant research results have yet been seen. The problem of network battery distribution in charging stations has been discovered by our previous research and is a typical TSP problem. Therefore, it can be solved by an intelligent algorithm such as an ant colony algorithm that solves the TSP problem. Ant colony algorithm Optimization, ACO), also known as the ant algorithm, is a probabilistic algorithm used to find the optimal path in the graph. Ant colony algorithm is a new type of global search group intelligence algorithm, which has been successfully used to solve the distribution network reconfiguration problem [22], multipoint routing problem [23] and document optimization distribution [24]. Based on the establishment of a mathematical model for the battery distribution of the charging station network, this problem can be solved by means of an ant colony algorithm.
(3) The overall optimization control of charging station network

During the demonstration run of the electric vehicle, there is a relatively fixed driving route. Therefore, there is no need to control the charging station, and the normal operation of the vehicle can also be guaranteed. However, when the vehicle runs on a large scale, traffic conditions and other reasons will cause the distribution of the charging vehicles in the charging station network to have strong randomness, which may result in the charging of a certain charging station being overcrowded, and the charging of another charging station is relatively free. Therefore, it is necessary and urgent to solve the optimization control problem of a large-scale and dynamic distributed system such as a charging station network.

At present, the research results on the control of charging stations have made certain progress, such as the control strategy of V2G smart charging stations for electric vehicles based on the TS fuzzy controller shown in [25], in which [26] elaborated on the electric vehicles in detail. The impact of the development of the "Vehicle to Grid (V2G)" technology on the grid. Literature [27] proposed a pricing strategy for charging and discharging electric vehicles. In the charge and discharge management strategy for electric vehicles, the document[28] proposed an optimized dispatching strategy based on convex relaxation for charging and discharging of electric vehicles. However, this method has a large amount of calculation and is real-time when a large number of electric vehicles are simultaneously connected. Poor. Literature [29,30] proposed a charging station control strategy based on Mamdani-type fuzzy controller. However, because the output of the Mamdani-type fuzzy system is not continuous, there is output blurring error. On the contrary, the TS-type fuzzy controller provides an accurate system equation, which brings convenience to the analysis and design of the system.

\section{CONCLUSIONS}

This essay discusses the control of large-scale electric vehicles charging station network. Firstly, main research contents of the current electric vehicle charging station are analyzed and summarized. Then, the key problems that need to be solved in the charging station network configuration and control process are pointed out. In addition, the corresponding solutions is proposed.

\section{ACKNOWLEDGMENT}

This work is supported by National Natural Science Foundation (NNCF) of China under Grant 61403124

\section{REFERENCES}

[1] Tian Meie.The necessity and trend of developing electric cars in China. Journal of Xi'an Shiyou University (Natural Science Edition),2010,Page:89-91.

[2] Zhang Xiang, Wang Jia and Yang Jianzhong,etc. Prospects of New Energy Vehicles for China Market, Hybrid and Eco-Friendly Vehicle Conference, 2008, Page:1-11.

[3] Mei Jie. study on power station planning for electric vehicle traffic properties. Master's thesis of Southeast University. 2017,U491.8;TM73.Page:11-16. 
[4] T.Winkler, P.Komarnicki and G. Mueller, etc. Electric vehicle charging stations in Magdeburg, IEEE Vehicle Power and Propulsion Conference, 2009, Page(s): 60 - 65 .

[5] YAO Jian-xin, WANG Mei, LUO Wei-ming. Construction and application of charging systems for electric mobiles. East China Electric Power, 2008, 36(8): 107-110.

[6] Zhao Wei,Jiang Fei and Xiao Yong,etc.Harmonic analysis of electric vehicle charging station in network ElectricPowerAutomationEquipment,2014,Page:61-66

[7] LU Yan-xia, ZHANG Xiu-min, PU Xiao-wen. Harmonic Study of Electric Vehicle Chargers. Proceedings of the CSU-EPSA, 2006, 18(3):5l-54.

[8] Niu Liyong, Jiang Jiuchun and Zhang Weige. A simulation model for analysis of current harmonics generated by charging stations for electric buses. CHINESE HIGH TECHNOLOGY LETTERS, 2008, 18(9): 953957.

[9] Huang Mei, Huang Shaofang. A Harmonic Engineering Calculation Method for Electric Vehicle Charging Station, Power System Technology, 2008, 32(20): 20-23.

[10] G.A.Putrus, P.Suwanapingkarl and D.Johnston,etc. Impact of electric vehicles on power distribution networks, IEEE Vehicle Power and Propulsion Conference, 2009, Page(s): 827-831.

[11] Yunyan Wang, Jingxin Li and Jiuchun Jiang, etc. Management information system of charging station for Electric Vehicle (EV), Proceedings of the Eighth International Conference on Electrical Machines and Systems, 2005, Vol.1, Page(s): 857 -860.

[12] Wang Jian, Jiang Jiuchun. The design and realization of the information managing system for the electrical vehicle charging station. Microcomputer Information, 2006, 22(15): 16- 17.

[13] Yan Hui, Li Gengyin and Zhao Lei, etc. Development of Supervisory Control System for Electric Vehicle Charging Station. Power System Technology, 2009, 33(12): 15-19.

[14] Wang Yunyan, Jiang Jiuchun and Niu Liyong. Management Information System of Electric Vehicle Charge-Station. Microcomputer Development, 2005, 15(11):57-59.

[15] Zhou Niancheng, Pu Songlin and Wang Qianggang, etc. Control strategy of energy storage buffer system for fast charging electric vehicle station. Power System Protection and Control, 2013, Page: 128-134.

[16] Huang Minli and Yu Aiqing.Study on Coordinated Charging Strategy for Battery Swapping Station Based on Improved Cuckoo Search Algorithm. Proceedings of the CSEE, 2018, Page: 1075-1084.

[17] YAO Jian-xin, WANG Mei, LUO Wei-ming. Construction and application of charging systems for electric mobiles. East China Electric Power, 2008, 36(8): 107-110.

[18] Kelly, Anthony: Decision Making Using Game Theory - An Introduction for Managers, Cambridge University Press, 2003,ISBN 0521-81462-6.

[19] Zhong Chong-xian Li Chun-guo Yang Lu-xi. Dynamic Resource Allocation Algorithm for Multi-cell OFDMA Systems Based on Noncooperative Game Theory. Journal of Electronics \& Information Technology, 2009, 31(8):1935-1940.

[20] YU Chun-hai, FAN Zhi-ping and ZHOU Wen-guang. Game TheoryBased Analysis of Trust During the Formation of R\&D Alliances Between Enterprises. Journal of Northeastern University (Natural Science), 2009, 30(10):1501-1503-1507.

[21] Kjell Hausken. Probabilistic Risk Analysis and Game Theory[J],Risk Analysis, 2002, 22(1):17-27.

[22] Chen Genjun, Wang Lei and Tang Guoqing. Distribution network reconfiguration for loss reduction using an ant colony optimization method. Proceedings of the CSU-EPSA, 2001,13(2): 48-53.

[23] Wang Ying and Xie Jianying. An Ant System Algorithm for Multicast Routing. Computer Engineering, 2001, 27(1):55-57.

[24] Xiong Zhi, Xiong Bu-yun and ZHOU Yin-dan. Document optimization distribution strategy based on ant colony algorithm for web server cluster. Computer Engineering and Design, 2009, 30(14):3389-3395.

[25] Yilmaz M, Krein P T. Review of the impact of vehicle-to-grid technologies on distribution systems and utility interfaces[J]. IEEE Transactions on Power Electronics, 2013, 28(12): 5673-5689.

[26] Liu Dongqi,Wang Yaonan and Shen Yongpeng. Research of V2G Smart Charging Station Control Strategy Using T-S Fuzzy Controller.Transactions of China ElectroTechnical Society,2016,Page:206-214.

[27] Jin Chenrui, Tang Jian, Ghosh P. Optimizing electric vehicle charging with energy storage in the electricity market[J]. IEEE Transactions on Smart Grid, 2013,4(1): 311-320.

[28] Wen C K, Chen J C, Teng J H, et al. Decentralized plug-in electric vehicle charging selection algorithm in power systems[J]. IEEE Transactions on Smart Grid, 2012, 3(4): 1779-1789.

[29] Thirugnanam K, Joy $\mathrm{T}$ P E R, Singh $\mathrm{M}$, et al. Modeling and control of contactless based smart charging station in V2G scenario[J]. IEEE Transactions on Smart Grid, 2014, 5(1): 337-348.

[30] Mukesh S, Praveen K, Indrani K. Implementation of vehicle to grid infrastructure using fuzzy logic controller[J]. IEEE Transactions on Smart Grid, 2012, 3(1): 565-577.

[31] Jia Yongji,Xing Fangfang.Location of Electric Vehicle Recharging Stations Based on Satisfactory Optimization.Journal of Donghua University (Natural Science), 2017.Page:739-746. 